\title{
Calcitriol Alleviates Hyperosmotic Stress-Induced Corneal Epithelial Cell Damage via Inhibiting the NLRP3-ASC-Caspase-I-GSDMD Pyroptosis Pathway in Dry Eye Disease
}

\author{
Jing Zhang (D)* \\ Yiqin Dai* \\ Yujing Yang \\ Jianjiang $\mathrm{Xu}$ (D)
}

Eye Institute and Department of Ophthalmology, Eye \& ENT Hospital, Fudan University; Shanghai Key Laboratory of Visual Impairment and Restoration; NHC Key Laboratory of Myopia, Fudan University, Shanghai,

People's Republic of China

*These authors contributed equally to this work
Correspondence: Jianjiang $\mathrm{Xu}$

Department of Ophthalmology and Visual Science, Eye, and ENT Hospital, Shanghai Medical College, Fudan University,

Shanghai, People's Republic of China

Email jianjiangxu@|26.com
Purpose: Inflammasome activation in response to elevated tear osmolarity behaves as an initial signal in dry eye-related corneal inflammation. Pyroptosis is another prominent consequence of inflammasome activation, which is featured by gasdermin D (GSDMD)driven cell lysis. This study aims to explore the role of pyroptosis in dry eye, and also to verify if calcitriol, a potential therapeutic agent for dry eye, has certain effects against hyperosmotic stress (HS)-induced pyroptosis in human corneal epithelial cells (iHCECs) and the underlying mechanism.

Methods: The expression of pyroptosis executor GSDMD in tears from dry eye patients was examined using western blotting. iHCECs were grown in hyperosmotic medium (450 mOsM) to mimic the feature of elevated tear osmolality of dry eye in vitro. Exogenous calcitriol or pyroptosis inhibitor disulfiram was used. The extent of pyroptosis of iHCECs under various treatments was examined by scanning electron microscopy, caspase- 1 and propidium iodide (PI) double staining by flow cytometry, immunofluorescent staining for ASC speck formation, and western blotting. Cell viability was measured by a CCK-8 assay and an LDH release assay.

Results: We found that pyroptosis was presented in dry eye patients, shown as the elevation of its effector GSDMD N-terminal domain (N-GSDMD) in patients' tears. Further in vitro results showed that HS promoted pyroptosis in human corneal epithelial cells, while exogeneous supplementation of disulfiram could reduce the number of iHCECs with pyroptotic markers. More importantly, we demonstrated that, in line with the effect of disulfiram, calcitriol could also alleviate HS-induced pyroptosis, through inhibiting the NLRP3-ASCcaspase-1-GSDMD pyroptosis pathway.

Conclusion: The current study provided direct evidence showing increased pyroptosis in dry eye patients. We demonstrated that calcitriol was able to effectively alleviate HS-induced corneal epithelial cell damage through inhibiting the NLRP3-ASC-caspase-1-GSDMD pyroptosis pathway. This study underlined calcitriol as a promising therapeutic agent for dry eye given its multiple therapeutic targets.

Keywords: calcitriol, dry eye, pyroptosis, GSDMD

\section{Introduction}

Dry eye (DE) is a common ocular disease characterized by a loss of homeostasis of tear film, resulting in ocular discomfort and even visual disturbance. ${ }^{1}$ Dry eye etiology is somehow complicated, while hyperosmolarity-triggered inflammation 
and damage of ocular surface are considered to play prominent roles. ${ }^{2}$ Increased tear osmolarity activates multiple stress signaling pathways in the ocular surface epithelium and resident immune cells, triggering the secretion of many innate inflammatory molecules that initiate a vicious self-perpetuating cycle. ${ }^{3}$ Inflammasomes are activated during this process, behaving as an initial signal to trigger subsequent inflammatory cascades. The wellstudied NLRP3 inflammasome interacts with apoptosisassociated speck-like protein (ASC), triggering caspase-1 activation, and further matures pro-inflammatory cytokines IL-1 $\beta$ and IL-18. ${ }^{4}$

Another prominent consequence of inflammasome activation is the induction of proinflammatory cell death called pyroptosis. ${ }^{5}$ Pyroptosis is featured by caspase-1/11induced gasdermin D (GSDMD)-driven cell lysis. Caspase-1, which processes pro IL-1 $\beta$ and IL-18 to their mature forms in the inflammasome pathway, can also cleave the pore-forming protein GSDMD to its active form N-GSDMD, leading to rupture of the plasma membrane and the release of intracellular contents, and finally cause local or systemic inflammatory responses. ${ }^{6,7}$ Pyroptosis has been recognized to participate in several ocular diseases, like diabetic retinopathy, ${ }^{8}$ acute glaucoma, ${ }^{9}$ and Aspergillus fumigatus keratitis. ${ }^{10}$ Very recently, Chen et al showed that GSDMD-driven pyroptosis was involved in desiccating stress-induced dry eye mice. ${ }^{11}$ So far, the link between dry eye and pyroptosis has been only established by using an experimental animal model, the direct evidence showing the involvement of pyroptosis in dry eye patients is still missing.

Calcitriol (1,25-dihydroxyvitamin D3), the active metabolite of vitamin D3, showed a promising therapeutic effect on clinical complains and ocular damage caused by dry eye. Our previous studies have already demonstrated that topical treatment of calcitriol could significantly ameliorate dry eye symptoms, resulting in increased tear volume, prolonged tear break up time (TBUT), and decreased fluorescein staining score and inflammatory index in benzalkonium chloride (BAC)-induced dry eye rats. ${ }^{12,13}$ We further showed that calcitriol could mitigate corneal inflammation at the initial stage, via suppressing NLRP3 activation and the following release of proinflammatory cytokines. ${ }^{14}$ However, it still remains unknown whether calcitriol has a certain effect on pyroptosis in dry eye scenario, another critical downstream consequence of NLRP3 inflammasome activation. Some hints emerged, as calcitriol or its precursor was shown to inhibit the pyroptosis signaling pathway in murine experimental autoimmune myocarditis, experimental colitis, or acute kidney injury, ${ }^{15-17}$ implying that the impact of calcitriol on epithelium pyroptosis in dry eye is worth further exploration. Therefore, the current study was designed to provide novel evidence of the correlation between pyroptosis and dry eye from the view of clinical perspective, and also to investigate the effect of calcitriol on dry eye-related epithelium pyroptosis and the underlying mechanisms.

\section{Materials and Methods}

\section{Participant Recruitment and Clinical Evaluation}

Fifteen dry eye patients (30 eyes) and fifteen normal controls (30 eyes) were recruited from the Eye and ENT hospital of Fudan University. The diagnosis of dry eye is made according to the following criteria: tear breakup time (TBUT) $\leq 10 \mathrm{~s}$, Schirmer's I test $\leq 5 \mathrm{~mm}$ for $5 \mathrm{~min}$, or corneal fluorescein staining (CFS) score $\geq 1$. The controls were individuals without any ocular disorders. The demographics and ocular sign scores of all participants are shown in Table 1. Tear samples were collected by Schirmer's I test without using local anesthesia. The wet parts of strips were cut into pieces, and then placed in $0.5 \mathrm{~mL}$ centrifuge tubes and stored at $-80{ }^{\circ} \mathrm{C}$ until use. The whole research procedure was performed following the Declaration of Helsinki and was approved by the Ethics Committee of the Eye and ENT Hospital of Fudan University (2019045). Written informed consent was obtained from all the participants.

Table I The Demographics and Ocular Sign Scores of All Subjects

\begin{tabular}{|l|c|c|c|c|c|}
\hline Group & Gender (M/F) & Age & TBUT(s) & CFS Score & Schirmer's I Test (mm) \\
\hline Control group & $5 / 10$ & $45.87 \pm 7.43$ & $10.69 \pm 1.83$ & $0.53 \pm 0.74$ & $6.98 \pm 1.56$ \\
\hline Dry eye group & $6 / 9$ & $43.33 \pm 7.99$ & $4.29 \pm 1.60$ & $2.13 \pm 0.83$ & $4.55 \pm 1.46$ \\
\hline
\end{tabular}

Note: Values are shown as mean \pm SD.

Abbreviations: TBUT, tear break up time; CFS score, corneal fluorescein staining. 


\section{Reagents and Antibodies}

Calcitriol was obtained from Sigma-Aldrich (St. Louis, MO, USA). Pyroptosis inhibitor, disulfiram (CAS No. 9777-8) and necrosulfonamide (CAS No. 1360614-48-7) were purchased from MedChem Express. All reagents were dissolved in DMSO or $100 \%$ molecular grade ethanol, and then diluted to desired concentration before use. The ultimate concentration of vehicle did not exceed $0.1 \%$. The untreated cells were grown in fresh medium containing $0.1 \%$ vehicle as a blank control. The primary antibodies against Gasdermin D, cleaved Caspase-1 (Asp297), IL-1 $\beta$ (D3U3E), and NLRP3 were obtained from Abcam (Cambridge, UK) or CST (Danvers, MA, USA). The secondary HRP-conjugated goat anti-rabbit IgG antibody was bought from Invitrogen (Carlsbad, CA, USA).

\section{Cell Culture}

The immortalized human corneal epithelial cell (iHCEC) line was provided by Prof. Wei-yun Shi, and cultured as previously described. ${ }^{12}$ Cells at passage $3-5$ were grown in hyperosmotic medium at $450 \mathrm{mOsM}$ through adding $100 \mathrm{mM} \mathrm{NaCl}$, treated with or without calcitriol.

\section{Extraction of Tear Proteins from Schirmer Strips}

Extraction of tear proteins from Schirmer strips were performed as previously described with some modifications. ${ }^{18}$ Briefly, RIPA lysis buffer (Beyotime, Shanghai, China) was added to the $0.5 \mathrm{~mL}$ centrifuge tube. After $30 \mathrm{~min}$, a puncture was made at the bottom of the tube and strip was secured inside. The $0.5 \mathrm{~mL}$ tube was then placed into a $1.5 \mathrm{~mL}$ tube, and centrifuged at $12,000 \mathrm{rpm}$ for $15 \mathrm{~min}$. The collected fluid from the strip was mixed with a loading buffer, boiled for $10 \mathrm{~min}$, and centrifuged. The samples were quantification by a BCA protein Assay kit (Beyotime, Shanghai, China) and then subjected to western blotting.

\section{Western Blotting}

Proteins were separated on an SDS polyacrylamide gel and transferred electronically to PVDF membranes. After 1 $\mathrm{h}$ blocking, the membranes were incubated with primary antibody (anti-Gasdermin D, CST \#96458; anti-cleaved Caspase1 (Asp297), CST \#4199; anti-IL-13 (D3U3E), CST \#12703; or anti-NLRP3, abcam, $\#$ ab214185) at $4{ }^{\circ} \mathrm{C}$ overnight. Then, the membranes were incubated with HRP-conjugated goat anti-rabbit IgG secondary antibody (Invitrogen \#31460) for
$1 \mathrm{~h}$. The bands were visualized by enhanced chemiluminescence solution (ECL, Thermo Scientific, USA). The results were pixels per band analyze using the ImageJ program.

\section{Cell Viability Assay}

Cell viability was detected by a CCK- 8 assay. Briefly, cells were grown in a 96-well plate at a density of $1 \times 10^{4}$ cells/ well and exposed to the various treatments. At the indicated time point, $10 \mu \mathrm{L}$ CCK-8 solution (Dojindo Laboratories, Kumamoto, Japan) mixed with $100 \mu \mathrm{L}$ culture medium were added to each well. After 2 $\mathrm{h}$ incubation, the absorbance was measured at $450 \mathrm{~nm}$ using a microplate reader (BioTek, USA).

\section{Lactate Dehydrogenase (LDH) Release Assay}

Increased release of $\mathrm{LDH}$ indicated the loss of plasma membrane integrity. Briefly, cells were grown in a 96-well plate for various treatments. Cell supernatant was harvested at the indicated time point, and LDH release was examined with an LDH assay kit (Beyotime, China) at absorbance of $490 \mathrm{~nm}$ with a microplate reader. The level of LDH release was counted as the percentage of the total amount, considered as the sum of the enzymatic activity present in the cellular lysate and that in the cell supernatant.

\section{Caspase-I and Propidium lodide (PI) Staining}

PI and the FAM-FLICA in vitro Caspase-1 Detection Kit (ImmunoChemistry Technologies, USA) were used to examine pyroptosis by flow cytometry following the manufacturer's instructions. Briefly, cells were incubated with the caspase- 1 detection probe (FAM-YVAD-FMK) at $37{ }^{\circ} \mathrm{C}$ in the dark. After $1 \mathrm{~h}$ incubation, cells were washed with cellular wash buffer to remove unbound FLICA reagent. Cells were stained with PI for an additional $10 \mathrm{~min}$, and then analyzed by flow cytometry (FACS Caliber; Becton Dickinson, Germany). Pyroptotic cells were defined as double positive for Caspase-1 and PI.

\section{Immunofluorescent Staining for ASC Speck Formation}

To visualize ASC speck formation in response to different treatments, cells cultured on 24-chamber slides were fixed with $4 \%$ PFA for $10 \mathrm{~min}$ and then permeabilized with $0.3 \%$ Triton X-100 for 15 min. Cells were then blocked 
with 3\% donkey serum and stained with anti-ASC (AdipoGen, AG-25B-0006) at $4{ }^{\circ} \mathrm{C}$ overnight. Then, Alexa-Fluor 555 conjugated secondary antibody was used. The slides were monitored using a fluorescence microscope (TS SPE; Leica Microsystems, Germany).

\section{Scanning Electron Microscopy}

At the indicated time point, cells were fixed with $3 \%$ glutaraldehyde for $2 \mathrm{~h}$, post-fixed for $30 \mathrm{~min}$ in $1 \%$ $\mathrm{OsO}_{4}$, and dehydrated in a graded concentration of ethanol. Then, the samples were critical-pointed dried, mounted on stubs, sputter coated with a thin layer of conductive metal, gold and palladium, and monitored by a scanning electron microscopy (JEM-6360LV, Japan).

\section{Statistical Analysis}

All in vitro experiments were performed independently at least three times. The data are presented as mean $\pm \mathrm{SD}$, and analyzed by one-way ANOVA or Student's $t$-test. $P<0.05$ was considered as statistically significant.

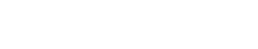

\section{Results}

Increased pyroptosis is observed in tears of dry eye patients and hyperosmotic stress (HS)-stimulated human corneal epithelial cells in vitro, while inhibition of pyroptosis by disulfiram protects cells against HS-induced cell cytotoxicity

To determine whether pyroptosis was involved in dry eye status, tears from dry eye patients were collected using Schirmer strips, and the expression of GSDMD, as well as its activated form N-GSDMD in tear proteins, were examined by western blotting. As shown in Figure 1, compared with normal controls, dry eye patients revealed a significant elevation of N-GSDMD expression $(0.34$ \pm 0.062 in controls vs $0.98 \pm 0.071$ in patients, $P=0.0025$ ), while the level of full length GSDMD (FL-GSDMD) was slightly increased in patients $(0.75 \pm 0.13$ in controls vs $0.84 \pm 0.10$ in patients, $P=0.6113$, Figure $1 C)$. The result indicated that activated pyroptosis was involved in dry eye status. Untreated Schirmer strip was shown as a blank control (Figure S1).

A

\begin{tabular}{|c|c|c|c|c|c|}
\hline \multicolumn{2}{|c|}{ group1 } & \multicolumn{2}{|c|}{ group2 } & \multicolumn{2}{|c|}{ group3 } \\
\hline Ns & Ps & Ns & Ps & Ns & Ps \\
\hline
\end{tabular}

N-GSDMD
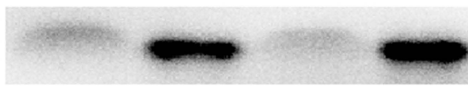

FL-GSDMD

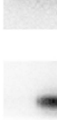



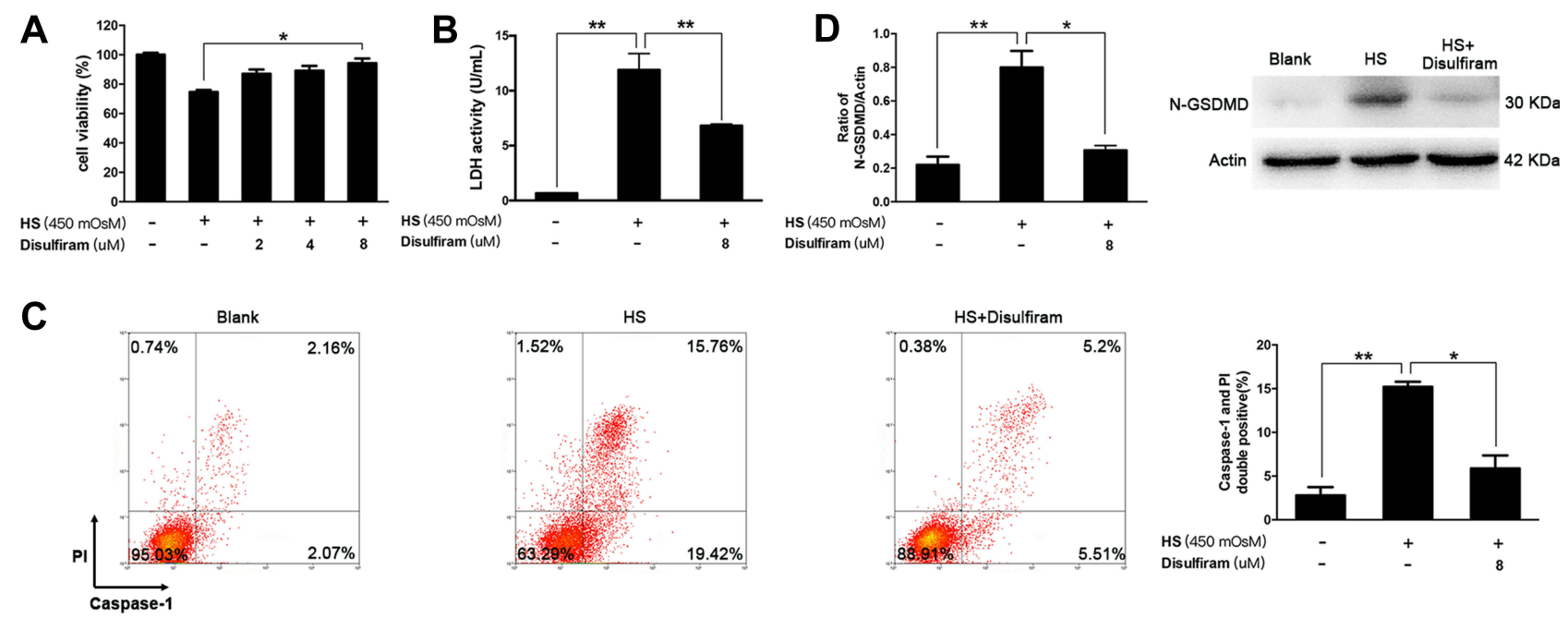

Figure 2 Increased pyroptosis was observed in hyperosmotic stress (HS)-triggered human corneal epithelial cells (iHCECs) in vitro, while inhibition of pyroptosis by disulfiram could protect cells from HS-induced cell cytotoxicity. (A) Cell viability of iHCECs treated with various concentrations of disulfiram (2, 4, $8 \mu$ M). (B) Disulfiram treatment $(8 \mu \mathrm{M})$ suppressed lactate dehydrogenase $(\mathrm{LDH})$ release in HS-stimulated cells. (C) Disulfiram treatment $(8 \mu \mathrm{M})$ reduced the proportion of pyroptotic cells, shown as Caspase-I/PI double positive by flow cytometry. (D) Relative protein level of N-GSDMD in blank, HS, and disulfiram treated groups. $* P<0.05$, $* * P<0.0$ I.

In the next setting, iHCECs were treated with hyperosmotic culture medium, to mimic the situation of elevated tear osmolality of dry eye in vitro. We found that the responses of iHCECs to hyperosmotic stress (HS) coincided with the features of activated pyroptosis (Figure S2). In particular, at $450 \mathrm{mOsM}$ hyperosmolar stress exposure, the percentage of pyroptotic cells (Caspase-1 and PI double positive) increased by 5.4-fold in comparison with untreated cells $(P=0.0075$, Figure 2C). The level of N-GSDMD showed 3.6-fold increment in HS-stimulated cells $(P=0.0033$, Figure 2D). However, such activation could be remarkably alleviated by disulfiram treatment. Disulfiram treatment led to a significant increase of cell viability, and also suppressed the release of LDH, a key indicator of membrane integrity $(P=0.036$ and 0.0015 , respectively, Figure 2A and B). More importantly, disulfiram treatment obviously suppressed HS-induced pyroptosis, shown as a $61.4 \%$ reduction in the proportion of pyroptotic cells, as well as a $61.7 \%$ reduction in the level of N-GSDMD (Figure 2C and D). The results indicated that HS could trigger iHCECs pyroptosis in vitro, while inhibition of pyroptosis by disulfiram could protect these cells against HS-induced cytotoxicity.

\section{Calcitriol Could Inhibit HS-Induced Pyroptosis in Human Corneal Epithelial Cells}

Calcitriol has been demonstrated to exert a protective role on dry eye-related inflammation cascades and cell apoptosis; here, we wondered whether it revealed certain effect on HS-induced pyroptosis. We found that calcitriol treatment could protect iHCECs against HS-induced plasma membrane damage, shown as decreased LDH release in the calcitriol-treated group (Figure 3A, $P=0.00018$ ). Much clearer evidence came from the observation under scanning electron microscopy, as the cell morphological changes represented the features of pyroptosis. As shown in Figure 3B, the surface of untreated cells was intact and exhibited tethered extracellular vesicles (EVs), while HS exposure caused the formation of membrane pits and pores of varying sizes, as well as a pronounced loss of EVs. However, such morphological changes could be remarkably reversed by calcitriol treatment. Furthermore, staining on pyroptotic cells confirmed the suppressive effort of calcitriol on HS-induced pyroptosis, shown as a $41.6 \%$ decrease of pyroptotic cells (Figure 3C, $P=0.0119$ ).

\section{Calcitriol Suppresses the Aggregation of ASC Speck Formation, and Inhibits NLRP3-ASC-Caspase-I-GSDMD Pyroptosis Pathway}

ASC speck formation is an important readout for NLRP3 inflammasome activation, further triggering subsequent cleavage of caspase-1 and GSDMD. To assess whether calcitriol affected this canonical pyroptosis pathway, immunofluorescence staining on the aggregation of ASC speck formation and quantification on the expression of 
A

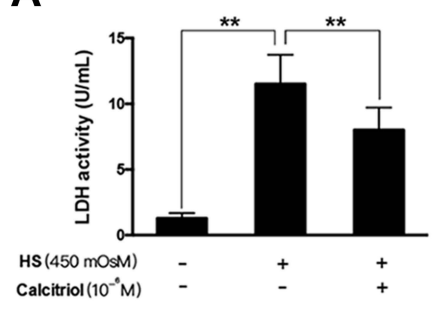

C

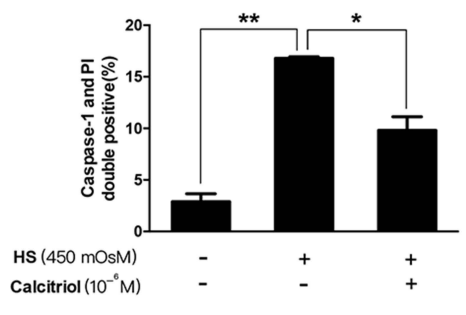

B
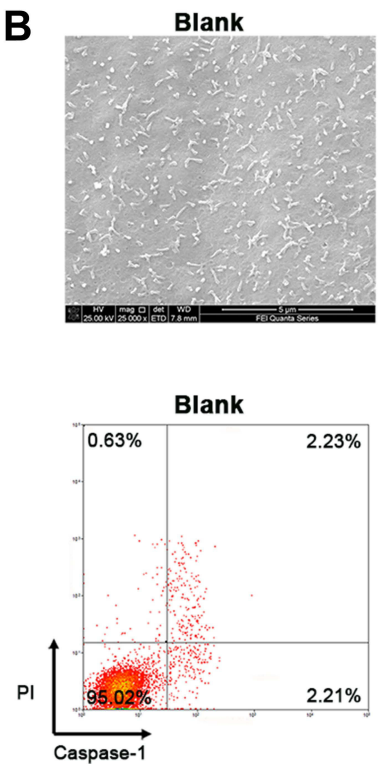

HS
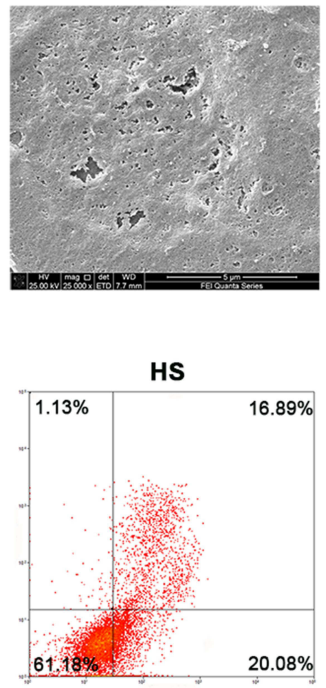

HS+Calcitriol
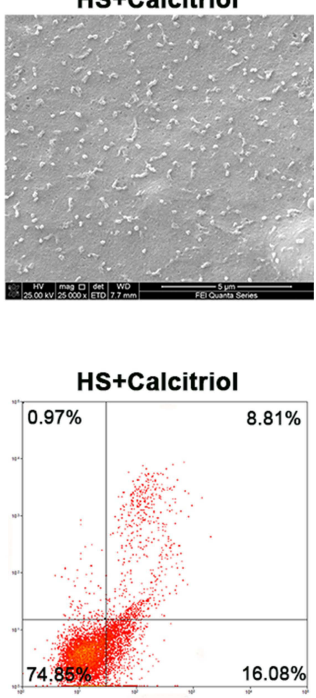

Figure 3 Calcitriol could inhibit HS-induced pyroptosis in human corneal epithelial cells. (A) Calcitriol treatment (I0 6 M) suppressed LDH release in HS-stimulated iHCECs. (B) Scanning electron micrographs of iHCECs from blank, HS, and calcitriol treated groups at $\times 25,000$ magnification. Scale=5 $\mu \mathrm{m}$. $(\mathbf{C})$ Calcitriol treatment $\left(10^{-6} \mathrm{M}\right)$ reduced the proportion of pyroptotic cells, shown as Caspase- I/PI double positive by flow cytometry. $* P<0.05, * * P<0.01$.

several other pyroptosis-related proteins were performed. As shown in Figure 4A, upon HS exposure, the aggregation of ASC was substantially increased when compared to the blank control. These ASC specks were around $2 \mu \mathrm{m}$ and aggerated in the paranuclear area of the cells, while calcitriol treatment caused a $66.8 \%$ reduction of the proportion of ASC specks $(P=0.0136)$. Furthermore, western blotting analysis showed that the expression of NLRP3, cleaved Caspase-1, N-GSDMD, and mature IL- $1 \beta$ in HSexposed cells were significantly higher than that of the untreated cells. In contrast, cells that co-treated with calcitriol revealed a remarkable decrease on the expression of these pyroptosis-related proteins, indicating that calcitriol showed a suppressive effect on the NLRP3-ASC-caspase1-GSDMD pyroptosis pathway (Figure 4B).

\section{Discussion}

Elevated tear osmolarity has been known as one of the core events in dry eye pathophysiology, inducing apoptosis of corneal and conjunctival cells and motivating inflammatory cascades that cause further cell death. ${ }^{19}$ Here, we found that another form of cell death, pyroptosis, was also presented in dry eye patients, shown as elevation of its executor N-GSDMD in patients' tears. Further in vitro results showed that hyperosmolarity promoted pyroptosis in human corneal epithelial cells, while exogeneous supplementation of a reported pyroptosis inhibitor, disulfiram ${ }^{20}$, could reduce the number of iHCECs with pyroptosis markers. More importantly, we demonstrated that, in line with the effect of disulfiram, calcitriol could also alleviate HS-induced pyroptosis, through inhibiting the NLRP3ASC-caspase-1-GSDMD pyroptosis pathway.

Pyroptosis is a highly inflammatory form of programmed cell death, using intracellularly generated pores to disrupt electrolyte homeostasis and thus leading cell death. ${ }^{21}$ Very recently, with an experimental murine model, Chen et al showed increased N-GSDMD level in corneal epithelium of mice exposed to desiccating stress (DS) when compared to the controls. The important role of GSDMD in dry eye pathogenesis was further confirmed using GSDMD-knock-out mice. ${ }^{11}$ In line with their results, here we demonstrated the involvement of pyroptosis in dry eye from the view of clinical angle, but provided more direct evidence demonstrating the elevation of its effector N-GSDMD in tears of dry eye patients. Although the methods of sample collection differed between the two studies, the observation of increased N-GSDMD was consistent, suggesting that pyroptosis might be ubiquitous in the corneal epithelium of dry eye, no matter whether in intact epithelial layer or detached cells within the tears.

The discovery of GSDMD as the final common step in pyroptosis and IL-1 $\beta / \mathrm{IL}-18$ release after inflammasome activation raised a novel approach for targeted therapy of dry eye. Indeed, the application of disulfiram, which was shown to block GSDMD pore formation, reduced the proportion of pyroptotic cells and prevented cells against HS-induced cytotoxicity. Interestingly, another Cystine-reactive compound, 
A
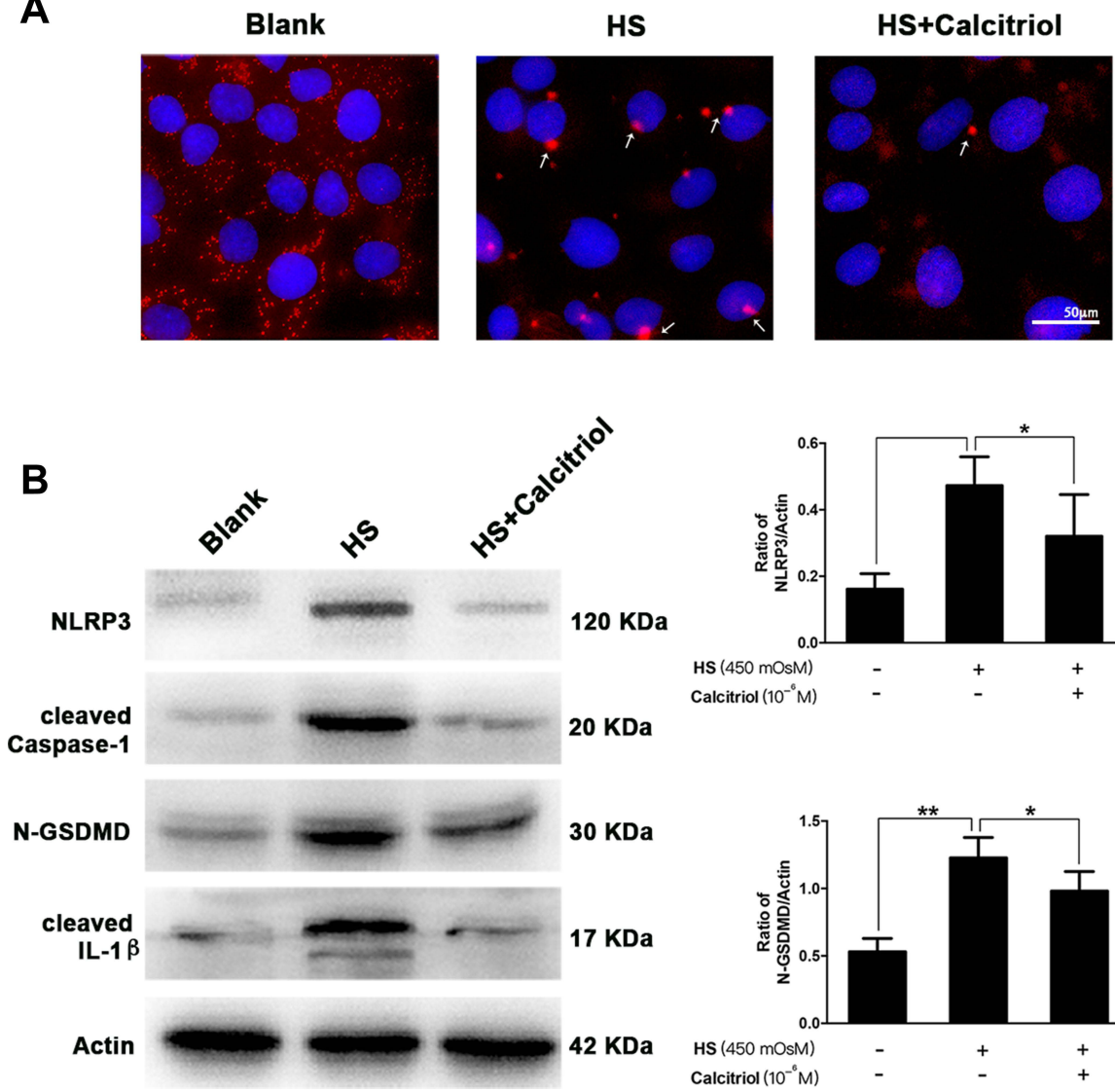

120 KDa

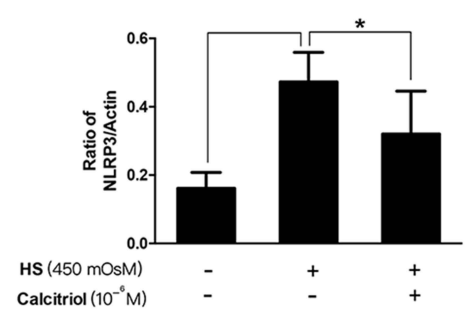

20 KDa

$30 \mathrm{KDa}$

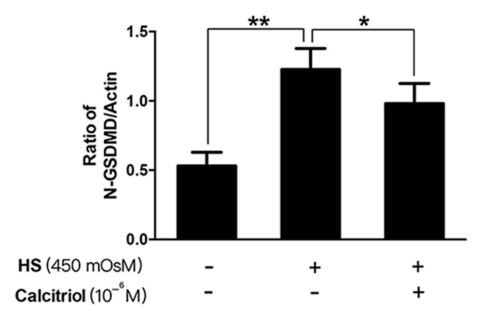

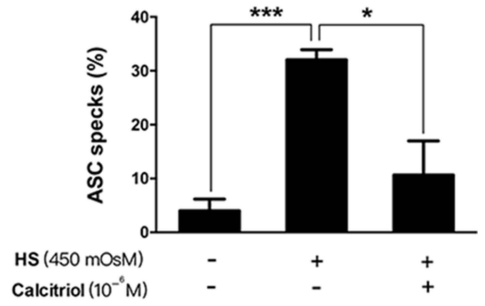
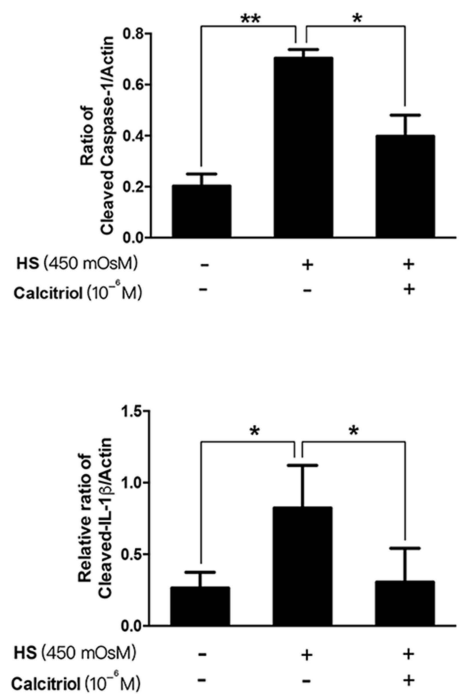

Figure 4 Calcitriol suppressed the aggregation of ASC speck formation, and inhibited the NLRP3-ASC-caspase-I-GSDMD pyroptosis pathway. (A) Immunofluorescence images of ASC speck formation (white arrows) in HS-stimulated iHCECs treated with/without calcitriol (ASC, red; nuclei, blue; $\times 200$ magnification). Analysis of three independent experiments assessing ASC speck formation to calculate the percentage of cells containing ASC speck. (B) The levels of pyroptosis-related proteins including NLRP3, cleaved Caspase-I, N-GSDMD, and cleaved IL-I $\beta$ were detected by western blotting, and quantification of the bands is shown in the right panel. $* P<0.05$, $* * P<0.0 \mathrm{I}$, $* * * P<0.001$.

necrosulfonamide (NSA), which has also been found to suppress lipopolysaccharide (LPS)-induced pyroptosis, ${ }^{22}$ revealed no protective effect on HS-treated corneal epithelial cells here (data not shown). The discrepancy implied that the potential difference in pyroptosis that triggered by HS or LPS required further elucidation. However, calcitriol behaved similarly to disulfiram, but exerted a much wider effect. Calcitriol apparently suppressed the aggregation of ASC speck formation, and also exerted a thorough inhibition on the proteolytic processing of a series of pyroptosis-related proteins. In combination with our previous publications that showed the inhibitory effect of calcitriol on corneal inflammation and cell apoptosis in dry eye, it is not surprising to deduce that calcitriol has pluripotent roles in alleviating ocular damage and clinical complaints caused by dry eye.

This study still has several limitations. First, quantitative measurement of the N-GSDMD level in tears was performed using mixed samples, given the limited number of detached cells that existed in the tears of each individual. However, the trend of elevated pyroptosis in the patients' group was evident, while whether it correlates to the severity of dry eye is worth further investigation. Secondly, although we confirmed that both pyroptosis and apoptosis were involved in hyperosmotic stress-induced corneal epithelial cell damage, it still remains unclear whether pyroptosis, apoptosis or other forms of cell death is the most responsible. Further study is in need to clarify this.

In summary, here we provided direct evidence showing increased pyroptosis in dry eye. We demonstrated that calcitriol was able to effectively alleviate HS-induced corneal epithelial cell damage through inhibiting the NLRP3ASC-caspase-1-GSDMD pyroptosis pathway. This study underlined calcitriol as a promising therapeutic agent for dry eye given its multiple therapeutic targets.

\section{Acknowledgment}

The authors were sponsored by National Natural Science Foundation of China $(81900819,81870630)$ and the 
Natural Science Foundation of Shanghai (17ZR1404400). The sponsor or funding organization had no role in the design or conduct of this research.

\section{Author Contributions}

$\mathrm{JZ}$ and JX designed the study; YD, JZ, and YY performed the experiments; JZ and YD analyzed the data; JZ wrote the original draft, YD, YY, and JX revised the manuscript. All authors contributed to data analysis, drafting or revising the article, have agreed on the journal to which the article will be submitted, gave final approval of the version to be published, and agree to be accountable for all aspects of the work.

\section{Disclosure}

The authors report no conflicts of interest in this work.

\section{References}

1. Craig JP, Nichols KK, Akpek EK, et al. TFOS DEWS II definition and classification report. Ocul Surf. 2017;15(3):276-283. doi:10.1016/j. jtos.2017.05.008

2. Pflugfelder SC, de Paiva CS. The pathophysiology of dry eye disease: what we know and future directions for research. Ophthalmology. 2017;124(11):S4-S13. doi:10.1016/j.ophtha.2017.07.010

3. Hessen M, Akpek EK. Dry eye: an inflammatory ocular disease. J Ophthalmic Vis Res. 2014;9(2):240-250.

4. Swanson KV, Deng M, Ting JP. The NLRP3 inflammasome: molecular activation and regulation to therapeutics. Nat Rev Immunol. 2019;19 (8):477-489. doi:10.1038/s41577-019-0165-0

5. Xue Y, Enosi Tuipulotu D, Tan WH, Kay C, Man SM. Emerging activators and regulators of inflammasomes and pyroptosis. Trends Immunol. 2019;40(11):1035-1052. doi:10.1016/j.it.2019.09.005

6. Shi J, Gao W, Shao F. Pyroptosis: gasdermin-mediated programmed necrotic cell death. Trends Biochem Sci. 2017;42(4):245-254. doi:10.1016/j.tibs.2016.10.004

7. He WT, Wan H, Hu L, et al. Gasdermin D is an executor of pyroptosis and required for interleukin-1beta secretion. Cell Res. 2015;25 (12):1285-1298. doi:10.1038/cr.2015.139

8. Homme RP, Singh M, Majumder A, et al. Remodeling of retinal architecture in diabetic retinopathy: disruption of ocular physiology and visual functions by inflammatory gene products and pyroptosis. Front Physiol. 2018;9:1268. doi:10.3389/fphys.2018.01268

9. Chen H, Deng Y, Gan X, et al. NLRP12 collaborates with NLRP3 and NLRC4 to promote pyroptosis inducing ganglion cell death of acute glaucoma. Mol Neurodegener. 2020;15(1):26. doi:10.1186/s13024020-00372-w

Journal of Inflammation Research

\section{Publish your work in this journal}

The Journal of Inflammation Research is an international, peerreviewed open-access journal that welcomes laboratory and clinical findings on the molecular basis, cell biology and pharmacology of inflammation including original research, reviews, symposium reports, hypothesis formation and commentaries on: acute/chronic inflammation; mediators of inflammation; cellular processes; molecular
10. Zhao W, Yang H, Lyu L, et al. GSDMD, an executor of pyroptosis, is involved in IL-1beta secretion in Aspergillus fumigatus keratitis. Exp Eye Res. 2021;202:108375. doi:10.1016/j.exer.2020.108375

11. Chen H, Gan X, Li Y, et al. NLRP12- and NLRC4-mediated corneal epithelial pyroptosis is driven by GSDMD cleavage accompanied by IL-33 processing in dry eye. Ocul Surf. 2020;18(4):783-794. doi:10.1016/j.jtos.2020.07.001

12. Zhang J, Dai Y, Wu D, Xu J. Calcitriol, the active metabolite of vitamin D3, inhibits dry eye related corneal inflammation in vivo and in vitro. Ocul Immunol Inflamm. 2019;27(2):257-265. doi:10.1080/ 09273948.2017.1372486

13. Lyu N, Zhang J, Dai Y, Xiang J, Li Y, Xu J. Calcitriol inhibits apoptosis via activation of autophagy in hyperosmotic stress stimulated corneal epithelial cells in vivo and in vitro. Exp Eye Res. 2020;200:108210. doi:10.1016/j.exer.2020.108210

14. Dai Y, Zhang J, Xiang J, Li Y, Wu D, Xu J. Calcitriol inhibits ROS-NLRP3-IL-1beta signaling axis via activation of Nrf2-antioxidant signaling in hyperosmotic stress stimulated human corneal epithelial cells. Redox Biol. 2019;21:101093. doi:10.1016/j. redox.2018.101093

15. Xiong Y, Lou Y, Su H, Fu Y, Kong J. Cholecalciterol cholesterol emulsion ameliorates experimental colitis via down-regulating the pyroptosis signaling pathway. Exp Mol Pathol. 2016;100 (3):386-392. doi:10.1016/j.yexmp.2016.03.003

16. Liu N, Su H, Zhang Y, Liu Z, Kong J. Cholecalciterol cholesterol emulsion attenuates experimental autoimmune myocarditis in mice via inhibition of the pyroptosis signaling pathway. Biochem Biophys Res Commun. 2017;493(1):422-428. doi:10.1016/j.bbrc.2017.09.006

17. Jiang S, Zhang $\mathrm{H}, \mathrm{Li} \mathrm{X}$, et al. Vitamin $\mathrm{D} / \mathrm{VDR}$ attenuate cisplatin-induced AKI by down-regulating NLRP3/Caspase-1/ GSDMD pyroptosis pathway. $J$ Steroid Biochem Mol Biol. 2020;206:105789. doi:10.1016/j.jsbmb.2020.105789

18. Posa A, Brauer L, Schicht M, Garreis F, Beileke S, Paulsen F. Schirmer strip vs. capillary tube method: non-invasive methods of obtaining proteins from tear fluid. Ann Anatomy. 2013;195 (2):137-142. doi:10.1016/j.aanat.2012.10.001

19. Luo L, Li DQ, Corrales RM, Pflugfelder SC. Hyperosmolar saline is a proinflammatory stress on the mouse ocular surface. Eye Contact Lens. 2005;31(5):186-193. doi:10.1097/01. ICL.0000162759.79740.46

20. Hu JJ, Liu X, Xia S, et al. FDA-approved disulfiram inhibits pyroptosis by blocking gasdermin D pore formation. Nat Immunol. 2020;21(7):736-745. doi:10.1038/s41590-020-0669-6

21. Liu X, Zhang Z, Ruan J, et al. Inflammasome-activated gasdermin D causes pyroptosis by forming membrane pores. Nature. 2016;535 (7610):153-158. doi:10.1038/nature18629

22. Rathkey JK, Zhao J, Liu Z, et al. Chemical disruption of the pyroptotic pore-forming protein gasdermin D inhibits inflammatory cell death and sepsis. Sci Immunol. 2018;3(26):eaat2738. doi:10.1126/ sciimmunol.aat 2738 